\title{
Building High Performance Teams through Collective Efficacy
}

\author{
Muhammad Zaki Rashidi \\ Dr. Fouzia Naeem Khan
}

\begin{abstract}
Today's organizations are changing their hierarchies due to the internal and external pressures and adapting innovative business processes; hence traditional functional pyramid and control is creating obstacles in handling sprouting issues and problems which require swift decision making. Teams are emerging as the basic unit of the organization to address this challenge and to enhance the productivity of the organizations by creating competitive edge achieved through teams' flexibility and agile working style. Understanding the very nature of the teams and the natural cohesion of its members is essentially important for the organizations to build result oriented and high performance teams. Social cognitive theory underpins the concept of understanding of human behavior through perceived self efficacy; and its extension group efficacy is presently focused in research to understand the team dynamics specially, the individuals constituting these teams. The realization of self and group efficacy or collective efficacy, leads to comprehend the composition of forces working in formation of a cohesive group. Collective efficacy also has established its relationship with performance and various research studies concluded that collective efficacy is vitally playing a role in group performance. Hence to predict the performance of a group, it is necessarily required to evaluate the collective efficacy of the group. This research is an attempt to find the relationships of self efficacy, collective efficacy with the performance of a team by taking into account the team behavior. The study was conducted with teams working on projects and the data collected established the fact that team behavior and collective efficacy are the fundamental requirements to build high performance teams. The research also provides an insight to measure them in a team.
\end{abstract}

Key words: Self efficacy, Collective efficacy, team building, group performance

\section{Introduction}

Globalisation and information technology have brought many radical changes to the organizations; these changes are not only reshaping the structure of the organization, but transforming the work-culture to be more independent, mobile, and demanding by dilating time and distance with greater reliance on the efficiency of their employees. The workforce is transforming from single performing individuals to the collaborating group of people and they are initiating, planning, coordinating and performing coherently to accomplish specific goals and responding the demanding challenges within and outside the organizations. Organizations are also restructuring their formal hierarchy,

Muhammad Zaki Rashidi is an MS student at SZABIST, zaki.rashidi@nu.edu.pk Dr. Fauzia Naeem Khan is Dean of Social and Media Sciences at SZABIST, Karachi.

Journal of Independent Studies and Research - MSSE

Volume 8

Number 1

January $2010 \mid 81$ 
and introducing innovative business processes blending with technology. They are bringing in the dynamic culture of cross functional teams to optimize their performance by reducing the cost. Efficient and effective teams are becoming significant units of the contemporary organization to face the upcoming and unanticipated challenges.

More emphasize is given to 'valuing difference' and 'managing diversity'. There can be many important dimensions of personal differences like: self concept, personality traits, skills and knowledge, personal values, and ethics. These factors provide a better understanding of a team if considered as an integrated package and gives the insight into organizational setup (McKenna, 2002; Singh, 2003).

Building a cohesive team of employees having different set of knowledge, skills, abilities and experiences is again a complex phenomenon on the part of organization. The performance of the team does not simply depend on the capabilities of the individuals' performances but more than that the collective performance of the group. In a successful team the members must have clear vision about the shared goals; a sense of commitment and loyalty; the ability to work in a cohesive manner; mutual accountability; timely access to needed resources and necessary skills to perform the subtasks; and swift coordination and communication (Lock \& Latham, 1990).

\subsection{Background}

According to (Haggbloom, Warnick, \& Warnick, 2002) Albert Bandura has been rated as the fourth most influential psychologist in the history of psychology as described by Judge et al (2007) for presenting his seminal theory, social cognitive theory. They further added that he is ranked among top five psychologists in terms of citation of his work (Knapp, 1985). His famous social cognitive theory revolves around the concept of self efficacy (Judge et al, 2007). Social cognitive theory (Bandura, 1986) posits that a person's level of motivation, affective states and action are based more on what they believe than on what is objectively the case (Siu et al, 2007). Bandura (1995) proposes that theory provides explicit guidelines that how to develop and enhance the quality of human functioning such as motivation and attainments.

Bandura (1994) defined self efficacy as, "people's belief about their capabilities to produce designated level of performance that exercise influence over events". Self efficacy refers to, "one's capabilities to organize and execute the course of action required to produce given attainment" (Bandura, 1997). The central debate of cognitive theory also regards the role of self efficacy beliefs in human functioning. Bandura (1997) presented his argument as, "people's level of motivation, affective states, and actions are based more on what they believe than on what is objectively true" (p.2). Industrial organizational psychology advocates that the most focal variable that has been related with self efficacy is performance; meta-analysis research also provides sufficient evidence (Stajkovic \& Luthans, 1998) that there is a strong link between self efficacy and performance of an employee. Lock and Latham (1990) as described by Vrugt and Koenis (2002) used the results of different studies and concluded that, "self efficacy contribute directly to the performance or indirectly via pursued goals". 
It is usually argues that self efficacy is culture dependent in some cultures self efficacy is high as compared to other cultures. Bandura $(1997,2002)$ suggested that 'sense of self efficacy' is universal. He further argued that, "there is cross-cultural commonality of agentic capacity rooted in beliefs of personal efficacy to produce effects results by one's actions, but the ways in which efficacy beliefs are developed and structured vary cross-culturally" (Siu et al, 2007).

There are three levels of self efficacy beliefs according to Gibbons and Weingart (2001). These levels are task specific, domain, and general self efficacy. Siu et al (2007) described that these three types differ in level of aggregation across tasks and performance domains and in ability over time and situation.

The belief of self efficacy can be developed through four major sources i.e. mastery experiences, vicarious experience provided by social models, social persuasion, and somatic and emotional states (Bandura, 1994). The most effective way of building self efficacy is mastery experience (Bandura, Adams, \& Beyer, 1977); success in this experience provides a strong belief in self and enhances the self efficacy. Whereas, failure undermine it especially if comes at early stage (Bandura, 1986, 1994). The second method of developing the self efficacy is through models which provide vicarious experiences. When one finds other people to perform a task successfully with their own similar conditions, then the belief in self efficacy is strengthened. Bandura $(1986 ; 1994)$ explained that "Perceived self efficacy is strongly influenced by perceived similarity to the models."Social persuasion is another factor that contribute in self efficacy, people who are persuaded verbally and encouraged to utilize their capabilities to master given activities mobilize their greater efforts and likely to sustain them as compared to the people have self doubts and receive negative comments about their capabilities. Finally, the fourth factor that is included in enhancement of self efficacy is somatic and emotional states that help in judging their capabilities to excel in their performance. Albert Bandura $(1977 ; 1986)$ notified that self efficacy is domain, task and situation specific. Self efficacy belief controls human performance through cognitive, motivational, affective and decisional process. (Bandura, 2002). The four major basis of self efficacy are not mutually exclusive but they are interdependent and interact in the process of selfevaluation (Bandura, 1986, 1994, 1997).

Our life is full of problems that we cannot handle alone, at organizational level different jobs are performed by a group of people, and their collective efforts produce the desired results. The strength of group lies not only in the self belief of the members to perform the task in hand but also in their collective efforts to put in and their belief perception as group to perform the task (Bandura, 1994).

The theory also takes into account that perceived collective efficacy represents the shared beliefs in the power to produce desired results by collective action (Bandura, 1997, 2000). Stajkovic \& Lee (2001) claimed that meta-analysis of various studies confirm that perceived collective efficacy enhances group functioning just as self efficacy enhances individual functioning. 
There are various fields where collective efficacy is used to measure and predict the performance of the group members associated with a certain community (FernandezBallesteros, Diez-Nicolas, \& Bandura, 2002); even the impact of perceived collective efficacy is being tested empirically in various fields through different experiments. These fields, as described by (Fernandez-Ballestros et al, 2002), include: education (Goddard, Hoy \& Hoy, 2004), athletics (Carron 1984; Spink, 1990; Mullen \& Cooper, 1994), combat games (Jex \& Bliese, 1999; Lindsley et al, 1994), and business (Gibson, 1995; Earley, 1994; Little \& Madigan, 1994).

Group efficacy measurement is still in its infancy and little agreement is available on this issue; however, researchers are emphasizing and proposing different methods to measure group efficacy (Bandura, 1997; Feltz \& Lirgg, 1998; Gibson, Randel \&Earley, 2000). Whiteoak, Laurence, and Hort (2004) presented a comparison of three methods to measure the collective efficacy of a group. The three methods are: (1) aggregate of self efficacy perceptions of individuals; (2) aggregate of individual's perception of group efficacy; and (3) consensual approach.

The first method suggest that, after collecting of individual's self efficacy if it aggregated then it will give group efficacy (Bandura; 1997, 2000). However, Lindsley, Brass, and Thomas (1995) criticized it and argued that individual's self efficacy would fail to take into account the dynamic social and organizational processes that occur within the group but Prussia and Kinicki (1996) favored this approach and accepted that it is line with the theoretical foundations.

Second method of measuring collective efficacy is averaging each individual's efficacy towards the performance and success of the group (Bandura; 1997, 2000). This approach is more popular and has greater acceptability among researchers (Earley, 1993; Durham et al, 2000; Goddard, 2002; Pescosolido, 2003).

Finally, the third and less popular approach is the use of 'group consensus responses' i.e. the group members together, by considering the group as an entity will determine its efficacy and $t$ his will be treated as the group efficacy (Gist, 1987). This approach has its pros and cons as discussed by Gibson et al. (2000).

Bandura (1997, pp 478-479) argues that, "in group context it is not possible to fully disentangle individual beliefs efficacy from beliefs about the capabilities of others". Whiteoak, Chalip, and Hort (2004) provided a detailed comparison of the three methods and set the criteria of reliability and validity to judge the superiority of one method to the other.

\subsection{Research Problem}

This study aims at identifying the self-efficacy, collective efficacy, team behaviour through behavioural observation scale. Also, it finds the key relationships between the variables: self efficacy, collective efficacy, team behaviour, task knowledge, and performance. Also different factors are considered which are essentially required for team work. 


\subsection{Significance}

Teams in organizations have become a common feature to cope with dynamic business environment and are essentially required for their survival and growth. Hence the study of effective team building through group efficacy will help the organizations to understand the basic characteristics required among employees to build and sustain high performance teams.

\subsection{Scope of the Study}

The study is limited to 12 groups of students which volunteered for this study; also data collection through the measurement scale is done only at one point of time.

\subsection{Variables}

- $\quad$ Self Efficacy

- $\quad$ Collective Efficacy

- Team Behaviour

- Task knowledge

- Team Performance

\section{Research Methodology}

\subsection{Research Design}

The study is based on a simulation of teams working in small groups for a project. A survey is conducted on these teams to collect the data after a period of six months of forming the teams. The data provided by the survey is analysed to measure the self efficacy and collective efficacy; team performance is measured after project completion through the supervisor of the group.

\subsection{Participants}

The participants of this research are undergraduate students of a private university. These students are currently studying in their final year and belong to computer science department. They are supposed to carry out their final year projects in groups of three to four members. They are supposed to develop a real life software project working in groups for a period of 10 months. The groups are formed by the choice of the students and their compatibility with their classmates. These compatibilities are usually developed through studying and working on different assignments during past three years of their studies. The group members often have diverse skills and knowledge sets with them, and do not necessarily belong to same stratum of CGPA. Hence their capabilities to perform different tasks in groups varied and required self management. 
The data is collected through three questionnaires, administered to measure the traits of self efficacy and collective efficacy among the groups. Three different types of efficacies are measured. First self efficacy, i.e. each group member is supposed to measure his or her own self efficacy to perform certain task in the group to accomplish the project activities. Second, is the peer evaluation i.e. each group member has evaluated its peers working in the same project to accomplish certain tasks assigned to them. Finally, the group efficacy, i.e. each team member is required to evaluate his or her group abilities as a whole to accomplish the tasks. The performance of the group also measured through mentors i.e. their supervisor, project manager, and project coordinator. Their task knowledge is measured through their CGPA, because it reflects the specific task knowledge the students have in the project domain.

\subsection{Measurement Scale}

The measurement scale used in this study comprises of 46 items, and 14 subscales. These subscales are used to measure the knowledge, skill and abilities (KSA). These fourteen subscales are: reaction to conflict ( 3 items), addressing to conflict (4 items), averts conflict ( 3 items), synthesis of team ideas ( 2 items), involving others ( 2 items), participation in problems solving (4 items), effective communication ( 3 items), goal setting achievement ( 2 items), team citizenship ( 6 items), commitment to team ( 2 items), focus on task in hand (6 items), preparation for meetings ( 2 items), providing feedback (4 items), and performance management ( 3 items). First seven factors belong to interpersonal and next seven factors are the part of self-management. The scale is borrowed from Taggar \& Brown (2001). They have shown that correlation between BOS and team performance is high and significant.

\subsection{Plan of Analysis}

Descriptive statistical analysis is used to calculate self efficacy and data is aggregated to computer collective efficacy. Correlations are used to determine the relationships between the factors of self efficacy, collective efficacy and performance.

\section{Analysis and Results}

\subsection{Descriptive Analysis}

The analysis of the data is conducted through correlation and descriptive statistics. Average of self efficacy, team behavior and collective efficacy of the 12 groups participated in this study is calculated in the following table. 
Table - 1: Self efficacy, Team behavior and collective efficacy

\begin{tabular}{|c|c|c|c|c|c|}
\hline Group & Self Efficacy & Team Behaviour & Collective Efficacy & Cumulative GPA & Performance \\
\hline 1 & 3.86 & 3.59 & 3.85 & 2.19 & 70 \\
\hline 2 & 3.92 & 3.92 & 3.86 & 3.47 & 75 \\
\hline 3 & 3.75 & 3.89 & 4.11 & 3.13 & 85 \\
\hline 4 & 4.07 & 4.01 & 3.97 & 2.89 & 70 \\
\hline 5 & 3.93 & 3.89 & 3.88 & 2.73 & 80 \\
\hline 6 & 4.21 & 4.14 & 4.09 & 2.49 & 90 \\
\hline 7 & 3.85 & 3.86 & 3.83 & 2.45 & 70 \\
\hline 8 & 3.78 & 3.94 & 3.89 & 3.28 & 80 \\
\hline 9 & 3.77 & 3.79 & 3.77 & 2.52 & 80 \\
\hline 10 & 4.13 & 3.89 & 3.99 & 2.46 & 65 \\
\hline 11 & 4.16 & 4.14 & 4.15 & 2.75 & 90 \\
\hline 12 & 3.79 & 3.74 & 3.9 & 2.47 & 80 \\
\hline
\end{tabular}

* CGPA is being treated as task knowledge.

\section{Analysis}

The self efficacy of some the groups is high as compared to other groups, however the maximum value of self efficacy is found 4.21 on a scale of 7 , which shows that the students working in the projects have low self efficacy on the average.

The team behaviour also varies from group to group and maximum value of the team behaviour is found 4.14, which is of the same group 6 . Hence students' contribution as a cohesive team work is less and some of the students work more as compared to other students.

There is vast difference in the collective efficacy of some the groups as compared to other groups. Group 3, 6 and 11 are showing high collective efficacy.

Self efficacy, team behaviour and collective efficacy are compared in this graph. This shows that except in first group, where team behaviour is low, in other groups team behaviour is above or equal to self efficacy and higher the team behaviour higher the collective efficacy.
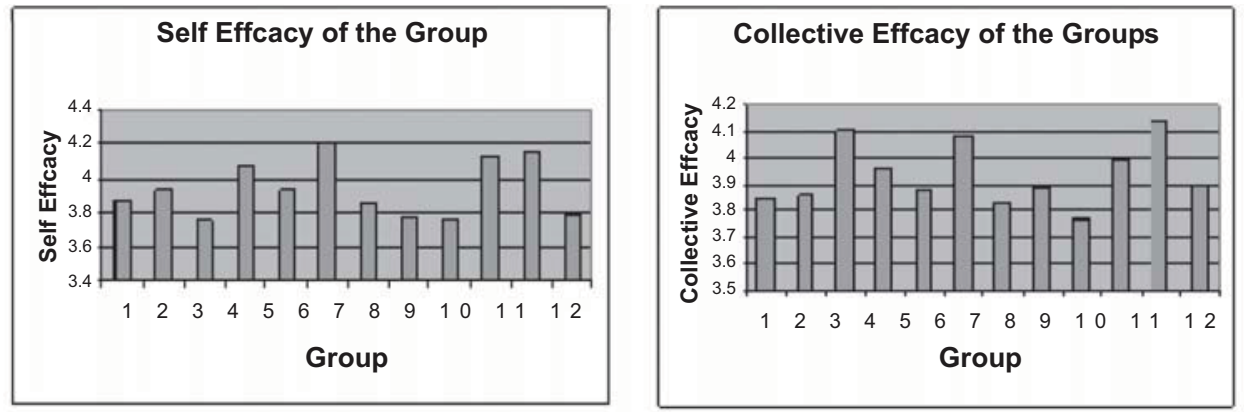

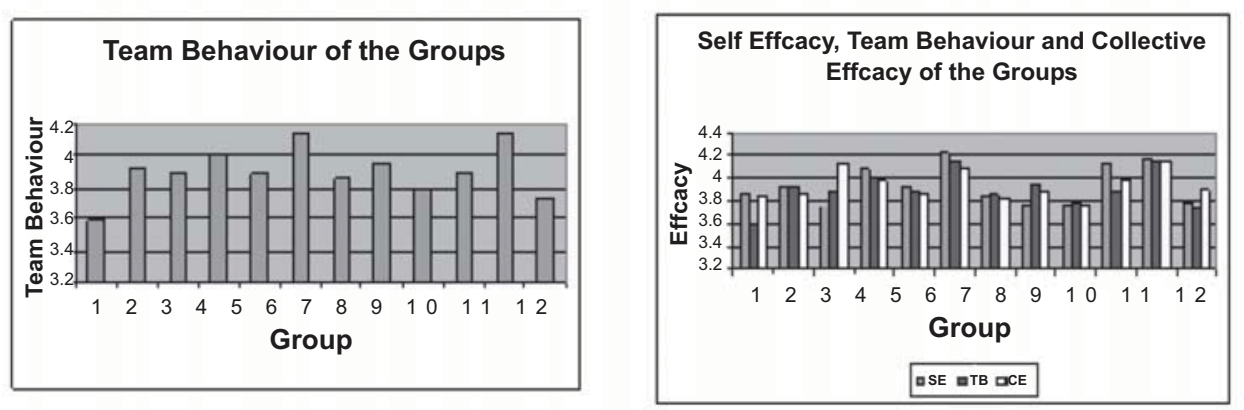

\subsection{Descriptive Analysis}

Table - 2: Descriptive analysis

\begin{tabular}{l|c|c|c}
\hline Variable & Mean & Standard Deviation & Range \\
\hline Self Efficacy & 3.94 & .46 & .16 \\
\hline Team Behavior & 3.90 & .55 & .15 \\
\hline Collective Efficacy & 3.94 & .38 & .11 \\
\hline Task Knowledge & 2.74 & .37 & 1.28 \\
\hline Performance & 77.91 & 7.76 & 25 \\
\hline
\end{tabular}

Self efficacy, team behaviour, and collective efficacy are on a scale of 1 to 7 , while task knowledge (i.e. CGPA) is on a scale of 0 to 4 , and performance is on a scale of zero to 100 .

\subsection{Correlation Analysis}

The correlation among the variables is calculated to see the relationship between them (table 3). The correlation between self efficacy, team behavior and collective efficacy is quite strong.

Table -3 : Correlation matrix of factors

\begin{tabular}{|c|c|c|c|c|c|}
\hline & SE & TB & CE & TK & PE \\
\hline SE & 1 & .694 & .588 & -.179 & .100 \\
\hline TB & .694 & 1 & .658 & .369 & .520 \\
\hline CE & .588 & .658 & 1 & .128 & .538 \\
\hline TK & -.179 & .369 & .128 & 1 & .200 \\
\hline PE & .100 & .520 & .538 & .538 & 1 \\
\hline
\end{tabular}

SE = Self Efficacy, TB = Team Behavior, CE = Collective Efficacy

$\mathrm{TK}=$ Task knowledge, $\mathrm{PE}=$ Performance Evaluation

The correlation matrix shows that there is a strong correlation between 'self efficacy' and 'team behavior', also there is a strong link between 'self efficacy' and 'collective efficacy'. Similarly 'team behavior' is also strongly related with 'collective efficacy' and 'performance'. There is negative correlation between self efficacy, and task knowledge, this can because both are evaluations are entirely different, first one is the average perception of the group, while second one evaluation of task knowledge through CGPA, or it is quite possible task knowledge cannot be measured through CGPA, for this other evaluation method is required. 


\subsection{Regression Analysis}

Multiple regression analysis also reveals that team behavior is a major contributor in performance of a group and next factor after this is collective efficacy. Self efficacy and task knowledge have negative coefficients; hence one interpretation of this may be that they do contribute directly in collective efficacy.

\subsection{Factor Analysis}

The factor analysis of the groups reveals that factors like: 'avert conflicts', 'commitment to teams', and 'focus on task in hand' have the lowest values in each group, while 'team citizenship' and 'involving others' have higher values across the groups as compared to other factors.

\section{Discussion}

Self efficacy, team behavior and collective efficacy are measured through BOS scale, where task knowledge is measured through CGPA and performance is given by respective project supervisor and project coordinator.

The data shows that wherever self efficacy is high, collective efficacy is also high and this confirms the initial idea presented that self efficacy contributes in collective efficacy. Also team behavior is playing pivot role in performance, where team behavior is obtained through peer evaluation of BOS scale. This means those groups where evaluation of each other is positive and higher on the scale are performing better as compared to those who do not rate higher each other. This also reveals the team conflict and lack harmony among team members. Hence we can safely say that higher the team behavior will enhance the performance of the team.

The graph of task knowledge shows less variation, hence it can be said that all the groups on the average have more or less similar task knowledge, but their performance is different, hence other factors besides task knowledge are contributing in team performance. These factors according to our analysis are team behavior and collective efficacy. This suggests having similar KSA even leads to better results if there is cohesion in the team.

In fact self efficacy and team behavior interacts and their interplay along with collective efficacy enhances the performance of a team. Various correlations and regression analysis also conform to this idea and strengthen the factor of team behavior as the key factor in performance management.

\section{Conclusion}

Self efficacy is a major factor contributing in collective efficacy and team behavior, while team behavior is pivot element to build the high performance teams. Organizations requiring result-oriented teams need to understand the self efficacy and collective 
efficacy of the group they are and this can be measured through behavior observation scale.

This study is very small scale study conducted through simulation on 12 groups of students, who already had their understanding with each other over the period of three years. Hence it is quite possible the estimates of team behavior and collective efficacy are affected by their behavior. Secondly they worked on different projects, which had different levels of difficulty, hence their performance is hampered if a group is undertaken a difficult project and team conflicts arises. Hence, if they have same project to work on then their performance can be measured and compared satisfactorily. Finally, their evaluation is conducted by their respective supervisors along with the project coordinator, according certain parameters. A possibility of variation in marks exists among the groups as well.

This study requires to be conducted on large number of groups working on identical tasks, having diverse people, with standard evaluation criteria. Hence their performance can be judged and better results can be drawn. Even comprehensive factor analysis may reveal various other factors among the groups and dominant factors which are involved in 'team behavior' can also be determined.

Still, there is less agreement among the researchers to measure the collective efficacy of the groups, this also requires an in depth analysis to develop a better scale and method to evaluate this. 


\section{References}

Bandura, A. (1977). "Towards a unifying theory of behavior change", Psychological Review, vol. 84, pp. 199 - 215.

Bandura, A. (1986). Social foundations of thought and action: A Social Cognitive Theory, Englewood Cliffs, Prentice-Hall, NJ.

Bandura, A. (1994). Self-efficacy. In V. S. Ramachaudran (Ed.), Encyclopedia of human behavior (vol.4, pp 71 -81). New York: Academic Press.

Bandura, A. (1995). Exercise of personal and collective efficacy in changing societies. In a Bandura (Ed.), Self efficacy in chainging societies, pp. 1-45, Cambridge University Press, NY.

Bandura, A. (1997). Self-efficacy: The exercise of control, W. H. Freeman Company, New York.

Bandura, A. (2000). "Health promotion from the perspective of social cognitive theory", in P. Norman, C. Abraham, \& M. Conner (Eds.), Understanding and changing health behavior: From health beliefs to self-regulation (pp. 299 -339). Amsterdam: Harwood Academic.

Bandura, A. (2002). Social cognitive theory in cultural context, Applied Psychology: An international Review, vol. 51, no.2, pp. 269 - 290.

Bandura, A., Adams, N. E., and Beyer, J. (1977). "Cognitive processes mediating behavioral change", Journal of Personality and Social Psychology, vol. 35, pp. 125 139.

Carron, A. V. (1984). Cohesion in sports teams. In J M. Silva, III \& R. S Weinberg (Eds.), Psychological foundations of sports (pp. 340 - 351). Champaign IL: Human Kinetics Publication.

Durham, C. C., Locke, E. A., Poon, J. M. L., and McLeod, P. L. (2000). Effects of group goals and time pressure on group efficacy, information seeking strategy, and performance, Human Performance, vol.13, pp.115 - 138.

Earley, P. C. (1993). East meets west meets Mideast: Further explorations of collectivistic and individualistic work groups, Academy of Management Journal, vol.36, pp.319 348.

Earley, P. C. (1994). "Self or group? Cultural effects of training on self efficacy and performance", Administrative Science Quarterly, vol. 39, pp.89 - 117.

Feltz, D.L. and Lirgg, C.D. (1998). "Perceived team and player efficacy in hockey",

Journal of Independent Studies and Research - MSSE

Volume $8 \quad$ Number 1

January $2010 \mid 91$ 
Journal of Applied Psychology, vol.83, pp. 557 - 564.

Fernandez-Ballesteros, R, Diez-Nicolas, J. and Bandura, A. (2002). Determinants and structural relation of personal efficacy to collective efficacy, Applied Psychology: An International Review, vol. 51, no.1, pp.107 - 125.

Gibbons, D. E. and Weingart, L. R. (2001). Can I do it? Will I try? Personal efficacy, assigned goals, and performance norms as motivators of individual performance, Journal of Applied Social Psychology, vol. 31, no. 3, pp. 624-648.

Gibson, C. B. (1995). Determinants and consequences of group-efficacy beliefs in work in work organizations in US, Hong Kong, and Indonesia. Doctoral dissertation, University of California, Irvine.

Gibson, C.B., Randel, A.E. \& Earley, P. C. (2000). "Understanding group efficacy: An empirical test of multiple assessment methods", Group \& Organization Management, vol. 25, pp. 67 - 97.

Gist, M.E. (1987). "Self-efficacy: Implications for organizational behavior and human resource management", Academy of Management Review, July, pp.472 - 485.

Goddard, R. D. (2002). "A theoretical and empirical analysis of the measurement of collective efficacy: The development of a short form", Educational and Psychological Measurement, vol.62, pp.97 - 110.

Goddard, R.D., Hoy, W.K. and Hoy, A.W. (2004). "Collective efficacy beliefs: The theoretical developments, empirical evidence, and future directions", Educational Researcher, vol. 33, no.3, pp. 3 - 13.

Haggbloom, S. J., Warnick, R., and Warnick, J. E. (2002). The 100 most eminent psychologist of the 20th century. Review of General Psychology vol. 6, pp. 139 - 152.

Judge, A. T., Jackson C. L., Shaw, J., Scott, B. A., and Rich, B. L. (2007). Self efficacy and work related performance: The integral role of individual differences, Journal of Applied Psychology, vol. 92, no.1, pp. 107 - 127.

Jex, S. M., and Bliese, P. D (1999). Efficacy beliefs as a moderator of the impact of work-related stressors: A multilevel study, Journal of Applied Psychology, vol.84, pp. $349-361$.

Knapp, T. J. (1985). Who is who in the American introductory psychology textbooks: a citation study. Teaching of Psychology, vol. 12, pp.15 - 17.

Lindsley, D.H., Brass, D.J., and Thomas, J.B. (1995). "Efficacy-Performance Spirals: A Multilevel Perspective", Academy of Management Review vol.20, pp.645 - 678.

Journal of Independent Studies and Research - MSSE

Volume 8

Number 1

January $2010 \mid 92$ 
Little B. L, and Madigan, R. M. (1994, Aug). "Motivation in work teams: A test of the construct of collective efficacy", paper presented at the annual meeting of the Academy of Management, Houston, TX.

Lock, E. A., and Latham, G. P. (1990). A theory of goal setting and task performance, Englewood Cliffs: Prentice-Hall.

McKenna, E. (2002). Business Psychology and Organizational Behaviour: A student handbook, (3rd ed.), Psychology Press, Sussex, UK.

Mullen, B. and Cooper, C. (1994). The relation between group cohesiveness and performance: An integration, Psychological Bulletin, vol. 115, pp. 210 - 227.

Pescosolido, A T. (2003). Group efficacy and group effectiveness: The efforts of groups efficacy over time on group performance and development, Small Group Research, vol. 34 , pp. 20 - 42.

Prussia, G. E. and Kinicki, A. J. (1996). A motivational investigation of group effectiveness using social-cognitive theory, Journal of Applied Psychology, vol. 81, pp. 187 - 198.

Singh N (2003). Organizational Behavior: Concepts, Theory, and Practices. Deep and Deep Publications Pvt. Ltd. New Delhi.

Siu, O. L., Lu, C. Q., and Spector, P. E. (2007). Employees' well being in greater China: The direct and moderating factor effects of general self-efficacy, Applied Psychology: An International Review, vol. 56, no. 2, pp. 288 - 301.

Spink, K. S. (1990). Group cohesion and collective efficacy of volleyball teams, Journal of Sport exercise Psychology, vol. 112, pp. 301-311.

Stajkovic, A. D. and Lee, D. S. (2001). A meta analysis of the relationship between collective efficacy and group performance. Paper presented at the national Academy of Management Meeting, August, Washington D.C.

Stajkovic, A. D. and Luthans, F. (1998). Self-efficacy and work-related performance: A meta-analysis, Psychological Bulletin, vol. 124, pp.240 - 261.

Taggar, S. and Brown T. C. (2001). Problem solving team behaviors development and validation of BOS and a hierarchical factor structure, Small Group Research, vol.32, no.6, pp. $698-726$.

Vrugt, A. and Koenis, S. (2002). Perceived self-efficacy, personal goals, social comparison, and scientific productivity, Applied Psychology: An international Review, vol. 51, no.4, pp.593-607.

Whiteoak, J. W., Chalip, L. and Hort, L.K. (2004). Assessing group efficacy comparing three methods of measurement, Small Group Research, vol. 35, no.2, pp. 158-173. 